\title{
Virus, allergic sensitisation and cortisol in infant bronchiolitis and risk of early asthma
}

\author{
Jon Olav Gjengstø Hunderi (1) 1,2,3, Leif Bjarte Rolfsjord (1) ${ }^{3,4}$, \\ Karin C. Lødrup Carlsen ${ }^{2,3}$, René Holst ${ }^{5}$, Egil Bakkeheim², \\ Teresa Løvold Berents ${ }^{3,6}$, Kai-Håkon Carlsen ${ }^{2,3}$ and Håvard Ove Skjerven ${ }^{2,3}$
}

Affiliations: ${ }^{1}$ Dept of Pediatrics and Adolescent Medicine, Østfold Hospital Trust, Grålum, Norway. ${ }^{2}$ Division of Pediatric and Adolescent Medicine, Oslo University Hospital, Oslo, Norway. ${ }^{3}$ Institute of Clinical Medicine, Faculty of Medicine, University of Oslo, Oslo, Norway. ${ }^{4}$ Dept of Pediatrics, Innlandet Hospital Trust, Elverum, Norway. ${ }^{5}$ Oslo Centre for Biostatistics and Epidemiology, Faculty of Medicine, University of Oslo, Oslo, Norway. ${ }^{6}$ Dept of Dermatology, Oslo University Hospital, Oslo, Norway.

Correspondence: Jon Olav Gjengstø Hunderi, Dept of Pediatrics and Adolescent Medicine, Østfold Hospital Trust, Postboks 300, 1714 Grålum, Norway. E-mail: jonhundso-hf.no

\section{ABSTRACT}

Background: Acute bronchiolitis during infancy and human rhinovirus (HRV) lower respiratory tract infections increases the risk of asthma in atopic children. We aimed to explore whether specific viruses, allergic sensitisation or cortisol levels during acute bronchiolitis in infancy increase the risk of early asthma, using recurrent wheeze as a proxy.

Methods: In 294 children with a mean (range) age of 4.2 (0-12) months enrolled during hospitalisation for acute infant bronchiolitis, we analysed virus in nasopharyngeal aspirates, serum specific immunoglobulin E against food and inhalant allergens, and salivary morning cortisol. These factors were assessed by regression analyses, adjusted for age, sex and parental atopy, for risk of recurrent wheeze, defined as a minimum of three parentally reported episodes of wheeze at the 2-year follow-up investigation.

Results: At 2 years, children with, compared to without, recurrent wheeze had similar rates of respiratory syncytial virus (RSV) (82.9\% versus $81.8 \%$ ) and HRV (34.9\% versus $35.0 \%)$ at the acute bronchiolitis, respectively. During infancy, $6.9 \%$ of children with and $9.2 \%$ of children without recurrent wheeze at 2 years were sensitised to at least one allergen $(p=0.5)$. Neither recurrent wheeze nor incidence rate ratios for the number of wheeze episodes at 2 years were significantly associated with specific viruses, high viral load of RSV or HRV, allergic sensitisation, or morning salivary cortisol level during acute bronchiolitis in infancy. Conclusion: In children hospitalised with acute infant bronchiolitis, specific viruses, viral load, allergic sensitisation and salivary morning cortisol did not increase the risk of early asthma by 2 years of age.

@ERSpublications

In infants with acute bronchiolitis, specific viruses including human rhinovirus, viral load and/or allergic sensitisation did not increase the risk of asthma by 2 years of age. http://bit.ly/2tCE9Yd

Cite this article as: Hunderi JOG, Rolfsjord LB, Carlsen KCLVirus, allergic sensitisation and cortisol in infant bronchiolitis and risk of early asthma. ERJ Open Res 2020; 6: 00268-2019 [https://doi.org/ $10.1183 / 23120541.00268-2019]$.

This article has supplementary material available from openres.ersjournals.com

The Bronchiolitis ALL study, SE Norway, is registered at www.clinicaltrials.gov with identifier number NCT00817466 and https://eudract.ema.europa.eu with identifier number 2009-012667-34. The data are stored on a research server at Oslo University Hospital. The data are also used for ongoing works and studies; therefore, they are not generally available. For discussion of data access, H.O. Skjerven may be contacted (e-mail: skjerven@gmail.com).

Received: 26 Sept 2019 | Accepted after revision: 27 Dec 2019

Copyright $\odot$ ERS 2020. This article is open access and distributed under the terms of the Creative Commons Attribution Non-Commercial Licence 4.0. 


\section{Introduction}

Acute bronchiolitis is the most common viral lower respiratory tract infection (LRTI) leading to hospitalisation in infants $[1,2]$, with respiratory syncytial virus (RSV) being the most commonly identified virus, followed by human rhinovirus (HRV) [3, 4]. Infants with moderate to severe bronchiolitis [5-9] and infants with atopic parents $[7,10]$ are reported to be at increased risk of developing recurrent wheeze and subsequent asthma. Both viral and host-specific factors may influence disease progression [6, 9]. A high genomic load of RSV has been associated with increasing disease severity [4, 11] but has not been well studied in relation to later wheeze and asthma.

Viral LRTI in children who are sensitised to allergens may increase the risk of childhood asthma development compared to LRTI in the absence of allergic sensitisation [10, 12]. A likely causal relationship between HRV in the presence of aeroallergen sensitisation and increased risk of wheeze, particularly at 1 year of age, has been suggested based upon a study among 285 high-risk children [13], while a Swedish cohort study reported that severe RSV bronchiolitis was a strong risk factor for allergic sensitisation later in childhood [14]. However, we are not aware of studies assessing the role of early allergic sensitisation in early infancy in risk of asthma, nor are we aware of allergic sensitisation being evaluated in terms for interactions with bronchiolitis for future asthma risk. We recently demonstrated that $8.4 \%$ of infants with acute bronchiolitis (4.2 months) were sensitised to at least on allergen [15].

The potential interaction between specific respiratory viruses and early allergic sensitisation in the development of asthma is therefore unclear, while studies assessing the role of allergic sensitisation and RSV/HRV infection in infants younger than 1 year for later asthma are largely lacking.

Increased levels of plasma and saliva cortisol during acute bronchiolitis [16, 17] may reflect the response to acute stress, while low cortisol levels are observed in children with asthma and allergic rhinitis [18, 19]. Stress, measured by serum cortisol levels, may therefore be involved in immune dysregulation, which may further increase airway inflammation through elevation of proinflammatory cytokines [20]. It is not clear if cortisol levels during acute bronchiolitis are associated with the subsequent development of asthma.

The aims of the present study were therefore to explore whether specific viruses, high viral load of RSV or $\mathrm{HRV}$, allergic sensitisation, or morning salivary cortisol in infants with acute bronchiolitis increase the risk of early asthma.

\section{Subjects and methods}

\section{Study design}

The present study included all children enrolled in the multicentre, randomised clinical Bronchiolitis ALL South-East Norway trial during hospitalisation for acute bronchiolitis in infancy, and who attended a 2-year follow-up study [21]. Briefly, 404 infants with moderate to severe acute bronchiolitis $<12$ months of age were enrolled at the time of admission to one of eight hospitals in southeast Norway in 2010 and 2011 [22]. Inclusion criteria included clinical signs of bronchiolitis [23] and a clinical score [24] of at least 4 on a scale from 0 to 10,10 indicating most severe disease (table S1). Infants with severe underlying disease, more than one previous episode of wheeze or 4 weeks persistent lower airway symptoms, or use of inhaled or systemic steroids in the last 4 weeks were excluded.

At enrolment, we performed a structured parental interview, and collected nasopharyngeal aspirates for viral analyses, blood samples for specific immunoglobulin E (s-IgE) analyses and morning salivary sample for cortisol analyses. The 2-year follow-up visit included parental structured interviews for detailed medical history and a clinical investigation.

Caregivers of all infants provided informed written consent at enrolment. The study was approved by the Regional Committees for Medical and Health Research Ethics and by the Norwegian Medicine Agency. The Bronchiolitis All South-East Norway study is registered at www.clinicaltrials.gov (NCT00817466) and EudraCT (2009-012667-34), and is registered in the Norwegian Biobank Registry. The study was audited by the Norwegian Medicines Agency in 2011

\section{Subjects}

The baseline characteristics of the 294 children who attended the 2-year follow-up visit were not significantly different from the 110 nonattendees, apart from a higher maternal $(\mathrm{p}<0.001)$ and paternal $(\mathrm{p}=0.019)$ education (table S2).

\section{Methods}

Structured interview by study physicians with caregivers at both visits addressed the previous and current health of the child and family members, parental atopy, socioeconomic factors and ethnicity, with a focus on episodes of bronchial obstruction (wheeze). Parental education was categorised into five levels; from 1 
(no school completed) to 5 (higher education, $>3$ years), with further details provided in the supplementary material.

Wheezing episodes were reported based upon the questions, "Has the child had episodes of wheezing and/ or chest tightness? If yes: specify the total number of episodes from birth to enrolment (first assessment) and from enrolment to the two-year assessment." Additionally, parents were asked if the child had doctor-diagnosed asthma.

Virus analyses including RSV and HRV, as well as HRV subtypes A/B and C, were performed using real-time PCR at the Dept of Allergy, University of Athens (Athens, Greece), as described in detail elsewhere [4]. As previously described in detail, high viral genomic load was identified using data-driven cluster analyses restricted to five clusters and classified as high versus all other concentration clusters per virus [4].

Blood samples were collected by venepuncture and analyses of s-IgE were performed at Fürst Medical Laboratory (Oslo, Norway) using ImmunoCAP (Phadia AB, Uppsala, Sweden). We first screened for allergic sensitisation with Phadiatop Infant, and in all samples proceeding with further analyses in all samples with serum s-IgE $\geqslant 0.15 \mathrm{kU} \cdot \mathrm{L}^{-1}(\mathrm{n}=89)$, for s-IgE to hen's egg white, cow's milk, peanut, cat, dog, birch, timothy and house dust mite. All analyses were performed in one batch and specified down to $0.10 \mathrm{kU} \cdot \mathrm{L}^{-1}$. Allergic sensitisation was defined as $\mathrm{s}-\mathrm{IgE} \geqslant 0.35 \mathrm{kU} \cdot \mathrm{L}^{-1}$ to at least one allergen. Results from analyses using the cut-off level of $0.1 \mathrm{kU} \cdot \mathrm{L}^{-1}$ are provided in the table S3 [15].

Salivary cortisol sampling was performed the first morning after hospital admission, as soon as possible after the child's awakening (after 06:00 h) and before the child's first meal, using two sorbettes (hydrocellulose; Salimetrics Europe Ltd, Suffolk, UK) as previously described in detail [25]. The sorbettes were then frozen at $-86^{\circ} \mathrm{C}$ until transferred to Karolinska Institutet (Stockholm, Sweden) for analysis by radioimmunoassay [17].

\section{Outcomes and potential explanatory variables}

The primary outcome was early asthma by use of the proxy recurrent wheeze at 2 years, defined as at least three episodes of wheeze, including the acute bronchiolitis at enrolment. We also used the total number of wheezing episodes by 2 years of age.

The main explanatory variables were: the presence of RSV, HRV, and HRV subtypes A/B and C in nasopharyngeal secretions; viral genomic load of RSV and HRV; allergic sensitisation to any allergen, any food allergen or any inhalant allergen, or to single allergens; as well as salivary morning cortisol level. Sex, age, parental atopy and time from birth to the time of the 2-year follow- up, hereafter referred to as exposure time, were used as covariates.

\section{Statistical analyses}

Continuous data were analysed using Students t-test and are presented as mean values with standard deviations, ranges or 95\% confidence intervals, where appropriate. Categorical data, analysed by Pearson Chi-squared test, are given as numbers and percentages.

The analyses focused on the total number of wheezing episodes experienced by 2 years of age, including the acute bronchiolitis at enrolment. A dichotomisation with at least three events was used as a proxy for early asthma and served as our primary outcome. It was analysed by a logistic regression.

A secondary analysis focused on the actual counts of wheezing episodes. According to the inclusion criterion, all children had at least one incidence and therefore a zero-truncated Poisson regression would be a natural candidate for this analysis. Due to overdispersion, the zero-truncated negative binomial model was, however, found more suitable. Both models used the same set of explanatory variables: the presence of RSV, HRV, and HRV subtypes A, B and C; viral genomic load of RSV and HRV; allergic sensitisation to any allergen, any food allergen or any inhalant allergen, or to single allergens as well as salivary morning cortisol level. Relevant interaction analyses were performed. Both models were adjusted for age, sex and parental atopy. The exposure time, given by the time from birth to the time of the 2-year follow-up, was used as an offset.

The significance level was set to 0.05 (5\%). Analyses were performed using IBM SPSS version 25.0 (IBM, Armonk, NY, USA.) and R 3.6.0 (The R Foundation, Vienna, Austria).

\section{Results}

Baseline and clinical characteristics at enrolment of the 294 children are shown in table 1 . The mean age (range) was $4.2(0-12)$ months at enrolment and 24.5 (9.7-35.2) months at the 2-year follow-up visit. 
The most commonly identified virus was RSV, in $82 \%$ of cases, with a high genomic load observed in $54 \%$; followed by HRV, identified in 35\% (table 1 and figure S1).

Allergic sensitisation was present in $8.1 \%$ of the infants, with food allergen sensitisation in $7.4 \%$ and $1.8 \%$ being sensitised to inhalant allergens. 13 infants (4.8\%) were monosensitised (table 1).

Salivary cortisol was available for 133 of the infants, with a geometric mean (95\% CI) of 42.0 (32.9-53.7) $\mathrm{mmol} \cdot \mathrm{L}^{-1}$.

TABLE 1 Characteristics at birth, study enrolment and 2 years of age are reported stratified by the presence or absence of recurrent wheeze at 2 years of age

\begin{tabular}{|c|c|c|c|}
\hline & Recurrent wheeze & No recurrent wheeze & p-value \\
\hline Participants & $143(48.6 \%)$ & $151(51.4 \%)$ & \\
\hline \multicolumn{4}{|l|}{ At birth } \\
\hline Male sex & $96 / 143(67.1 \%)$ & $85 / 151(56.3 \%)$ & 0.06 \\
\hline GA weeks & $38.6 \pm 2.4$ & $38.5 \pm 4.0$ & 0.95 \\
\hline Born at $\mathrm{GA}<37$ weeks & $14 / 110(12.7 \%)$ & $15 / 116(12.9 \%)$ & 0.96 \\
\hline Birth weight g & $3347 \pm 632$ & $3497 \pm 642$ & 0.05 \\
\hline \multicolumn{4}{|l|}{ At enrolment } \\
\hline Age days mean (range) & $134(14-348)$ & $115(7-363)$ & 0.05 \\
\hline Weight g & $6650 \pm 1886$ & $6245 \pm 1794$ & 0.06 \\
\hline Eczema & $15 / 131(11.5 \%)$ & $14 / 140(10.0 \%)$ & 0.70 \\
\hline One previous episode of wheeze & $34 / 127(26.8 \%)$ & $35 / 133(26.3 \%)$ & 0.93 \\
\hline Length of hospital stay $h$ & $85 \pm 72$ & $76 \pm 59$ & 0.16 \\
\hline Need of supportive treatment & 73/142 (51.4\%) & $79 / 150(52.7 \%)$ & 0.83 \\
\hline \multicolumn{4}{|l|}{ Virus detected during acute bronchiolitis } \\
\hline RSV & $107 / 129(82.9 \%)$ & $112 / 137(81.8 \%)$ & 0.80 \\
\hline HRV & $45 / 129(34.9 \%)$ & 48/137 (35.0\%) & 0.98 \\
\hline HRV A or B & $12 / 129(9.3 \%)$ & $16 / 137(11.7 \%)$ & 0.53 \\
\hline HRV C & $33 / 129(25.6 \%)$ & $32 / 137(23.4 \%)$ & 0.67 \\
\hline RSV high genomic load & $68 / 129(52.7 \%)$ & $77 / 137(56.2 \%)$ & 0.57 \\
\hline HRV high genomic load & $8 / 129(6.2 \%)$ & $8 / 137(5.8 \%)$ & 0.90 \\
\hline More than one virus & $80 / 129(62.0 \%)$ & $90 / 137(65.7 \%)$ & 0.53 \\
\hline \multicolumn{4}{|l|}{ Allergic sensitisation" } \\
\hline Any sensitisation & $9 / 130(6.9 \%)$ & $13 / 141(9.2 \%)$ & 0.49 \\
\hline Any food sensitisation & $8 / 130(6.2 \%)$ & $12 / 141(8.5 \%)$ & 0.46 \\
\hline Any inhalant sensitisation & $3 / 130(2.3 \%)$ & $2 / 141(1.4 \%)$ & 0.59 \\
\hline Egg sensitisation & $5 / 130(3.8 \%)$ & $3 / 141(2.1 \%)$ & 0.40 \\
\hline Cow's milk sensitisation & $4 / 130(3.1 \%)$ & $9 / 141(6.4 \%)$ & 0.20 \\
\hline Peanut sensitisation & $1 / 141(0.7 \%)$ & $2 / 130(1.5 \%)$ & 0.52 \\
\hline Polysensitisation & $4 / 130(3.1 \%)$ & $3 / 141(2.1 \%)$ & 0.62 \\
\hline Monosensitisation & 4/130 (3.1\%) & $9 / 141(6.4 \%)$ & 0.20 \\
\hline Cortisol $\mathrm{mmol} \cdot \mathrm{L}^{-1}$ geometric mean $(95 \% \mathrm{Cl})$ & $37.0(30.2-45.4)$ & $33.7(28.5-39.8)$ & \\
\hline \multicolumn{4}{|l|}{ At the 2-year follow-up } \\
\hline Age days mean (range) & 747 (291-1055) & 725 (368-979) & 0.07 \\
\hline Asthma diagnosed by physician & $51 / 143(35.7 \%)$ & $5 / 151(3.3 \%)$ & $<0.001$ \\
\hline Asthma medication used & $108 / 134(80.6 \%)$ & $28 / 142(19.7 \%)$ & $<0.001$ \\
\hline \multicolumn{4}{|l|}{ Parental education } \\
\hline Maternal education" & $3.93 \pm 0.94$ & $4.05 \pm 1.03$ & 0.31 \\
\hline Paternal education" & $3.76 \pm 0.97$ & $3.92 \pm 1.02$ & 0.21 \\
\hline \multicolumn{4}{|l|}{ Parental allergic diseases } \\
\hline $\mathrm{Any}^{+}$ & $70 / 128(54.7 \%)$ & $60 / 136(44.1 \%)$ & 0.09 \\
\hline Maternal asthma & $22 / 112(19.6 \%)$ & $14 / 123(11.4 \%)$ & 0.08 \\
\hline Paternal asthma & $16 / 112(14.3 \%)$ & $15 / 123(12.2 \%)$ & 0.64 \\
\hline Maternal rhinoconjunctivitis & $21 / 124(16.9 \%)$ & $21 / 134(15.7 \%)$ & 0.78 \\
\hline Paternal rhinoconjunctivitis & $31 / 124(25.0 \%)$ & $25 / 134(18.7 \%)$ & 0.22 \\
\hline Maternal eczema & $20 / 128(15.6 \%)$ & $11 / 135(8.1 \%)$ & 0.06 \\
\hline Paternal eczema & $15 / 128(11.7 \%)$ & $10 / 135(7.4 \%)$ & 0.23 \\
\hline \multicolumn{4}{|l|}{ Environment } \\
\hline Smoking at home & $19 / 124(15.3 \%)$ & $19 / 129(14.7 \%)$ & 0.90 \\
\hline
\end{tabular}


At the 2-year follow-up investigation, $49 \%$ had recurrent wheeze, while doctor-diagnosed asthma was reported for $56(19 \%)$ children. The mean \pm SD number of wheeze episodes was $5.25 \pm 7.4$ at 2 years of age and $49 \%$ had used at least one asthma medication during the last year (table 1).

Recurrent wheeze by 2 years of age was neither significantly associated with RSV or HRV, nor with rates of high viral load of RSV or HRV during acute infant bronchiolitis (figure 1a and table 1). The rate of $\mathrm{HRV} \mathrm{C}$ at the time of acute bronchiolitis was similar in children with recurrent wheeze $(25.6 \%)$ and without recurrent wheeze $(23.4 \%)(\mathrm{p}=0.7)$.

Allergic sensitisation at enrolment was not significantly different among infants with (7.4\%) compared to those without recurrent wheeze $(9.2 \%)(\mathrm{p}=0.49)$ (figure $1 \mathrm{~b})$. Similar results were observed using analyses with cut-off values of allergic sensitisation of $0.10 \mathrm{kU} \cdot \mathrm{L}^{-1}$ (table S3).

Morning salivary cortisol levels in infancy were similar among children who, at 2 years of age, had recurrent wheeze and children who did not (geometric mean (95\% CI) 33.7 (28.5-39.8) versus 37.0 (30.2-45.4) $\mathrm{nmol} \cdot \mathrm{L}^{-1}$, respectively) (figure $1 \mathrm{c}$ ).

In multivariate analyses adjusted for age, sex, parental atopy and time since enrolment, recurrent wheeze at 2 years of age was not significantly associated with specific viruses, high viral load of RSV or HRV, allergic sensitisation, or morning salivary cortisol level in infancy (table 2). We found no significant interactions between specific viruses, viral load sensitisation and salivary cortisol levels.

The zero-truncated negative binomial regression analysis showed no significant effects upon the incidence rate ratios for recurrent wheeze of any specific viruses, high viral load of RSV or HRV, allergic sensitisation, or morning salivary cortisol level (table 3). No significant interactions between specific viruses, viral load sensitisation and salivary cortisol levels were further identified.

\section{Discussion}

Recurrent wheeze at 2 years of age was not significantly associated with specific viruses, high viral load of RSV or HRV, allergic sensitisation, or morning salivary cortisol during acute bronchiolitis leading to hospitalisation in the first year of life.

The lack of associations between recurrent wheeze at 2 years of age and type of virus identified during the acute bronchiolitis in infancy in our study is supported by a Swedish and Norwegian study reporting that the risk of asthma and recurrent wheeze at follow-up in early childhood was independent of the presence of RSV during acute bronchiolitis [9,26]. Furthermore, recurrent wheeze at 5 years of age was similar among HRV and RSV associated wheeze during infancy in 198 high-risk infants in an Australian birth cohort [10]. In contrast, a recent review [5] and others [7, 27] suggests that HRV-induced LRTI increase future asthma risk compared to other viruses, in line with a suggestion that the epithelial barrier function in the airways may be impaired with HRV infection, initiating an airway inflammatory response [28]. The apparent differences in associations between HRV versus RSV and later wheeze in our, compared to an Italian [29] and a Finnish study [30], may be related to differences in the detected viruses at the acute bronchiolitis. While we found HRV in 6\%, RSV in 53\% and both in $29 \%$ (figure S1), the Italian study of 230 infants using nasal washes identified HRV in 9\%, RSV in $43 \%$ and both in $0.4 \%$, and the Finnish study of 329 infants found the corresponding rates of $11 \%, 63 \%$ and $3 \%$ in nasopharyngeal aspirates, respectively. Thus, the analytic sensitivity of viral detection or the viral profile among the populations may influence the likelihood of detecting risk differences for later recurrent wheeze. Furthermore, the young age of our study population compared to infants older than 1 year may reflect different pathophysiological mechanisms by which LRTI increases the risk of future asthma. The bronchiolitis cohort studied by SiguRs et al. [14] included infants with RSV bronchiolitis only and healthy controls. This may have contributed to a misconception that increased risk of asthma development was specific for RSV bronchiolitis.

The lack of association between HRV C and recurrent wheeze at 2 years of age in the present study is in contrast to the increased risk of later hospitalisation for respiratory illness in HRV C positive infants [31], and increased severity of acute asthma in children with HRV C infections compared to HRV A and B [32].

The finding in the present study that high RSV and HRV genomic loads were not significantly associated with subsequent recurrent wheeze at 2 years of age is, to the best of our knowledge, novel. A high viral load appears to cause more severe bronchiolitis, as shown in the Bronchiolitis All study and by others [4, 11 , but we are not aware of studies investigating the association between viral load during acute infection and later recurrent wheeze and asthma.

Our finding that allergic sensitisation in early infancy was not associated with early asthma does not support the suggestion that early recognition of s-IgE sensitisation against common food and aeroallergens may identify wheezing children who are at risk of asthma development [33]. To the best of our 

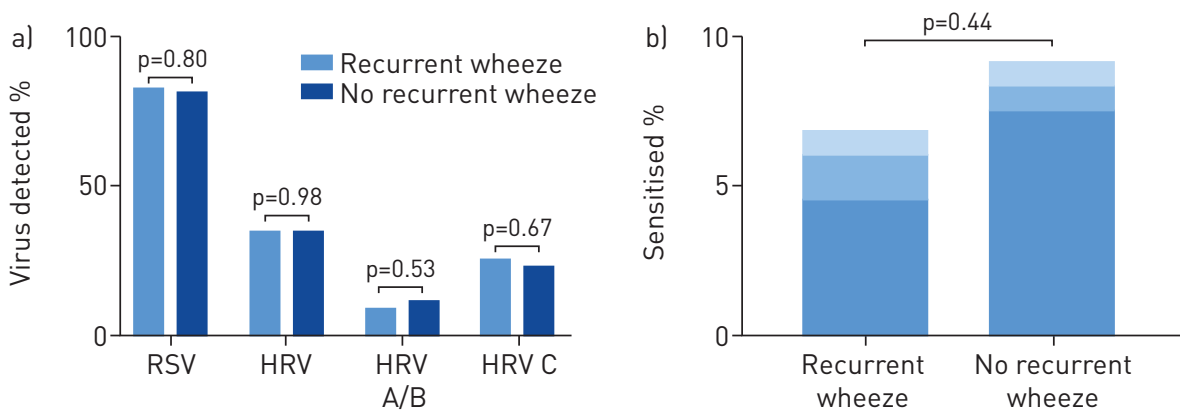

Sensitisation to any inhalant allergen (s-lgE $\geqslant 0.35 \mathrm{kU} \cdot \mathrm{mL}^{-1}$ )

Sensitisation to inhalant and food allergen (s-lgE $\geqslant 0.35 \mathrm{kU} \cdot \mathrm{mL}^{-1}$ )

Sensitisation to any food allergen

(s-lgE $\geqslant 0.35 \mathrm{kU} \cdot \mathrm{mL}^{-1}$ )

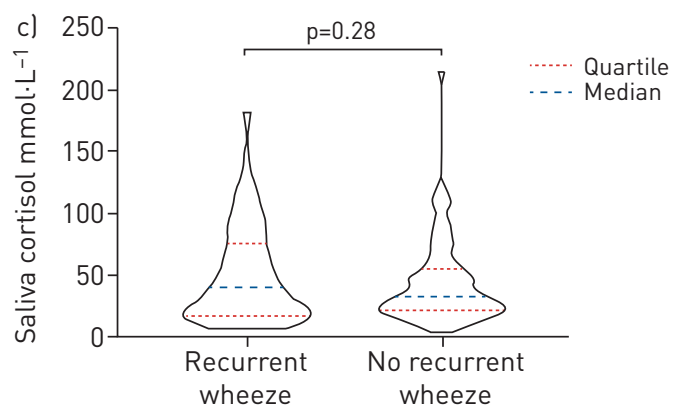

FIGURE 1 a) The distribution of respiratory syncytial virus (RSV), human rhinovirus (HRV), and HRV species A and $B$, and C; b) the distribution of sensitisation to inhalant and food allergens; and c) the distribution of morning saliva cortisol sampled the first morning after hospitalisation, in infants hospitalised with acute bronchiolitis at study enrolment (0-12 months of age, mean age 4.2 months), compared to children with recurrent wheeze and no recurrent wheeze at 2-year follow-up. s-IgE: specific immunoglobulin E.

knowledge, this is the first study to explore whether allergic sensitisation at the time of acute infant bronchiolitis increases the risk of recurrent wheeze. Our results are supported by a community-based study of 263 high-risk children that failed to show that a positive skin-prick test at 6 months of age increased the risk of recurrent wheeze at 2 years of age, while persistent wheeze at 5 years was associated with sensitisation first at 2 years of age [10]. Conversely, in a Finnish randomised controlled trial enrolling 3-23-month-old children with their first episode of wheeze, allergic sensitisation at a mean age of

\section{TABLE 2 The odds ratios of having recurrent wheeze at 2 years of age are shown by factors} observed among infants (0-12 months of age) who were admitted to hospital with acute

\section{bronchiolitis}

Recurrent wheeze OR $(95 \% \mathrm{CI})$

p-value

Viral detection during acute bronchiolitis
RSV
HRV
HRV A or B
HRV C
RSV high genomic load
HRV high genomic load
Multiple viruses
Allergic sensitisation
Any sensitisation
Any food sensitisation
Any inhalant sensitisation
Egg sensitisation
Cow's milk sensitisation
Peanut sensitisation
Polysensitisation
Salivary morning cortisol

$0.91(0.45-1.83)$

$1.03(0.59-1.78)$

0.78

$0.74(0.31-1.8)$

$1.19(0.65-2.19)$

$0.84(0.50,1.42)$

$0.93(0.30-2.84)$

$0.83(0.47-1.44)$

0.93

0.51

0.58

0.52

0.90

$0.72(0.28-1.89)$

$0.71(0.25-1.96)$

0.51

$1.08(0.19-20.89)$

$1.33(0.27-6.45)$

$0.41(0.10-1.64)$

1.00 (0.99-1.01)

Bivariate odds ratios are adjusted for sex, age at inclusion and parental atopy. RSV: respiratory syncytial virus; HRV: human rhinovirus. ${ }^{\#}$ : specific immunoglobulin $\mathrm{E} \geqslant 0.35 \mathrm{kU} \cdot \mathrm{L}^{-1}$. 


\begin{tabular}{|c|c|c|}
\hline & Recurrent wheeze IRR $(95 \% \mathrm{CI})$ & p-value \\
\hline \multicolumn{3}{|c|}{ Viral detection during acute bronchiolitis } \\
\hline RSV & $0.95(0.70-1.28)$ & 0.86 \\
\hline HRV & $0.72(0.57-0.91)$ & 0.16 \\
\hline HRV A or B & $0.58(0.40-0.83)$ & 0.14 \\
\hline HRV C & $0.84(0.65-1.09)$ & 0.51 \\
\hline RSV high genomic load & $0.92(0.73-1.15)$ & 0.70 \\
\hline HRV high genomic load & $0.61(0.38-0.99)$ & 0.31 \\
\hline Multiple viruses & $0.91(0.72-1.16)$ & 0.69 \\
\hline \multicolumn{3}{|l|}{ Allergic sensitisation ${ }^{\#}$} \\
\hline Any sensitisation & $1.46(0.97-2.19)$ & 0.36 \\
\hline Any food sensitisation & $1.53(1.0-2.36)$ & 0.32 \\
\hline Any inhalant sensitisation & $1.18(0.48-2.90)$ & 0.85 \\
\hline Egg sensitisation & $3.58(1.82-3.58)$ & 0.059 \\
\hline Cow's milk sensitisation & $0.49(0.29-0.95)$ & 0.17 \\
\hline Peanut sensitisation & $1.45(0.25-8.33)$ & 0.83 \\
\hline Polysensitisation & $1.44(0.60-3.49)$ & 0.68 \\
\hline Salivary morning cortisol & $1.01(1.0-1.0)$ & 0.56 \\
\hline
\end{tabular}

11 months was associated with increased risk of asthma at 8 years of age [34]. Like our study, most children in the Finnish study were admitted to hospital. However, the rate of allergic sensitisation in our study was $8 \%$ with only $2 \%$ being sensitised to inhalant allergens, while $17 \%$ in the Finnish study were sensitised to any allergen and 5\% to inhalant allergens. As previously shown in this cohort [15], infants older than, compared to younger than, 4 months were significantly more often sensitised to allergens. Thus, the young age of the infants in the present study probably represents a time at which most infants have not started to develop s-IgE to relevant allergens increasing the risk of asthma. In the COAST (Childhood Origins of Asthma) birth cohort study [13], high-risk children sensitised to aeroallergens were at increased risk of wheezing illness caused by HRV, but s-IgE was not analysed before 1 year of age.

No significant interaction was observed between HRV bronchiolitis and allergic sensitisation for recurrent wheeze in the present study. Our novel finding contrasts with the increased risk of asthma observed among children with HRV wheezy illness who were sensitised to allergens, compared to those that were not $[10,13]$. The contradictory result may suggest that the underlying mechanisms in acute bronchiolitis may differ from those in older children or may be related to a difference in hereditary risk in the study populations $[7,10,13]$. Our study does, therefore, not support the hypothesis that recurrent wheeze, which is triggered by HRV infection in susceptible infants, is conferred by early allergic sensitisation [10, 12].

Apart from ours, few studies have assessed the risk of recurrent wheeze related to potential subgroups of bronchiolitis; younger infants with predominantly RSV, a phenotype of HRV in atopic infants and bronchiolitis due to other virus [35]. Clearly, more studies are needed to provide further insight into the role of early allergic sensitisation and viral LRTI in asthma development.

We found no association between cortisol level in infants hospitalised with acute bronchiolitis and recurrent wheeze at 2 years of age, in contrast to the lower saliva cortisol level observed in children with asthma [18]. Previous results in this cohort showed that infants with acute bronchiolitis had higher levels of morning salivary cortisol than controls at enrolment, but similar cortisol levels at 2 years of age [17].

The present study is strengthened by the prospective design with a 2 -year follow up study in a cohort of well-characterised infants hospitalised with acute bronchiolitis. The rate of recurrent wheeze of $47 \%$ is in line with others $[7,10,36,37]$. The study was based on physician-led interviews instead of self-reported questionnaires. Parentally reported wheezing episodes may not, in all cases, have been documented by a physician, which is a potential limitation in this study. However, $>80 \%$ of the children with, versus $20 \%$ without, recurrent wheeze reported to have used asthma medication, supporting the use of recurrent wheeze as a proxy for early asthma. A Swedish study, initially reported by WENNERGRen et al. [9], reported increased risk of asthma maintained up to 27 years of age [38] 
Although the size of the study population is reasonable in relation to similar studies, the low number of children with allergic sensitisations in this age group limits the ability of the present study to detect significant interactions with HRV or RSV. A longer follow-up period could have provided further insight into the role of early sensitisation in early viral infection and asthma development.

In conclusion, in children hospitalised with acute bronchiolitis during the first year of life, specific viruses and viral load, allergic sensitisation, and salivary morning cortisol did not increase the risk of early asthma. It remains unclear to what degree allergic sensitisation in early infancy may contribute to early asthma development after viral LRTI.

Acknowledgments: We thank all children and their caregivers for their participation, and we are grateful for the contribution of the principal investigators and their colleagues in the paediatric departments at the eight hospitals in the southeast of Norway: Innlandet Hospital Trust, Elverum and Lillehammer; Vestre Viken Hospital Trust, Drammen; Vestfold Hospital Trust; Telemark Hospital Trust, Skien; Sørlandet Hospital Trust, Kristiansand; Oslo University Hospital Trust; and Østfold Hospital Trust. The study was performed within ORAACLE (the Oslo Research Group of Asthma and Allergy in Childhood; the Lung and Environment).

Conflict of interest: J.O.G. Hunderi has nothing to disclose. L.B. Rolfsjord has nothing to disclose. K.C.L. Carlsen received payment for a presentation during EAACI 2018 from Thermo Fisher Scientific. R. Holst has nothing to disclose. E. Bakkeheim has nothing to disclose. T.L. Berents reports service on an advisory board for Sanofi and a lecture for Perrigo, outside the submitted work. K-H. Carlsen has nothing to disclose. H.O. Skjerven has nothing to disclose.

Support statement: The study received support in part by Medicines for Children, Pediatric Dept, Haukeland University Hospital, Bergen, Norway. The first author received funding from Østfold Hospital Trust for this project. Funding information for this article has been deposited with the Crossref Funder Registry.

\section{References}

1 Wright AL, Taussig LM, Ray CG, et al. The Tucson Children's Respiratory Study. II. Lower respiratory tract illness in the first year of life. Am J Epidemiol 1989; 129: 1232-1246.

2 Meissner HC. Viral bronchiolitis in children. N Engl J Med 2016; 374: 1793-1794.

3 Mansbach JM, Piedra PA, Teach SJ, et al. Prospective multicenter study of viral etiology and hospital length of stay in children with severe bronchiolitis. Arch Pediatr Adolesc Med 2012; 166: 700-706.

4 Skjerven HO, Megremis S, Papadopoulos NG, et al. Virus type and genomic load in acute bronchiolitis: severity and treatment response with inhaled adrenaline. J Infect Dis 2016; 213: 915-921.

5 Jartti T, Gern JE. Role of viral infections in the development and exacerbation of asthma in children. J Allergy Clin Immunol 2017; 140: 895-906.

6 Martinez FD, Morgan WJ, Wright AL, et al. Diminished lung function as a predisposing factor for wheezing respiratory illness in infants. N Engl J Med 1988; 319: 1112-1117.

7 Lemanske RF Jr. Jackson DJ, Gangnon RE, et al. Rhinovirus illnesses during infancy predict subsequent childhood wheezing. J Allergy Clin Immunol 2005; 116: 571-577.

8 Sigurs N, Aljassim F, Kjellman B, et al. Asthma and allergy patterns over 18 years after severe RSV bronchiolitis in the first year of life. Thorax 2010; 65: 1045-1052.

9 Wennergren G, Hansson S, Engstrom I, et al. Characteristics and prognosis of hospital-treated obstructive bronchitis in children aged less than two years. Acta Paediatr 1992; 81: 40-45.

10 Kusel MM, de Klerk NH, Kebadze T, et al. Early-life respiratory viral infections, atopic sensitization, and risk of subsequent development of persistent asthma. J Allergy Clin Immunol 2007; 119: 1105-1110.

11 DeVincenzo JP, El Saleeby CM, Bush AJ. Respiratory syncytial virus load predicts disease severity in previously healthy infants. J Infect Dis 2005; 191: 1861-1868.

12 Holt PG, Upham JW, Sly PD. Contemporaneous maturation of immunologic and respiratory functions during early childhood: implications for development of asthma prevention strategies. J Allergy Clin Immunol 2005; 116 : $16-24$.

13 Jackson DJ, Evans MD, Gangnon RE, et al. Evidence for a causal relationship between allergic sensitization and rhinovirus wheezing in early life. Am J Respir Crit Care Med 2012; 185: 281-285.

14 Sigurs N, Bjarnason R, Sigurbergsson F, et al. Respiratory syncytial virus bronchiolitis in infancy is an important risk factor for asthma and allergy at age 7. Am J Respir Crit Care Med 2000; 161: 1501-1507.

15 Skjerven HO, Gjengsto Hunderi JO, Carlsen KH, et al. Allergic sensitisation in infants younger than one year of age. Pediatr Allergy Immunol 2019; 31: 103-206.

16 Pinto RA, Arredondo SM, Bono MR, et al. T helper 1/T helper 2 cytokine imbalance in respiratory syncytial virus infection is associated with increased endogenous plasma cortisol. Pediatrics 2006; 117: e878-e886.

17 Rolfsjord LB, Bakkeheim E, Berents TL, et al. Morning salivary cortisol in young children: reference values and the effects of age, sex, and acute bronchiolitis. J Pediatr 2017; 184: 193-198.

18 Bakkeheim E, Mowinckel P, Carlsen KH, et al. Reduced basal salivary cortisol in children with asthma and allergic rhinitis. Acta Paediatr 2010; 99: 1705-1711.

19 Landstra AM, Postma DS, Boezen HM, et al. Role of serum cortisol levels in children with asthma. Am J Respir Crit Care Med 2002; 165: 708-712.

20 Priftis KN, Papadimitriou A, Nicolaidou P, et al. Dysregulation of the stress response in asthmatic children. Allergy 2009; 64: 18-31.

21 Skjerven HO, Rolfsjord LB, Berents TL, et al. Allergic diseases and the effect of inhaled epinephrine in children with acute bronchiolitis: follow-up from the randomised, controlled, double-blind, Bronchiolitis ALL trial. Lancet Respir Med 2015; 3: 702-708. 
22 Skjerven HO, Hunderi JO, Brugmann-Pieper SK, et al. Racemic adrenaline and inhalation strategies in acute bronchiolitis. N Engl J Med 2013; 368: 2286-2293.

23 Court SD. The definition of acute respiratory illnesses in children. Postgrad Med J 1973; 49: 771-776.

24 Kristjansson S, Lodrup Carlsen KC, Wennergren G, et al. Nebulised racemic adrenaline in the treatment of acute bronchiolitis in infants and toddlers. Arch Dis Child 1993; 69: 650-654.

25 Tryphonopoulos PD, Letourneau N, Azar R. Approaches to salivary cortisol collection and analysis in infants. Biol Res Nurs 2014; 16: 398-408.

26 Fjaerli HO, Farstad T, Rod G, et al. Acute bronchiolitis in infancy as risk factor for wheezing and reduced pulmonary function by seven years in Akershus County, Norway. BMC Pediatr 2005; 5: 31 .

27 Lehtinen P, Ruohola A, Vanto T, et al. Prednisolone reduces recurrent wheezing after a first wheezing episode associated with rhinovirus infection or eczema. J Allergy Clin Immunol 2007; 119: 570-575.

28 Looi K, Troy NM, Garratt LW, et al. Effect of human rhinovirus infection on airway epithelium tight junction protein disassembly and transepithelial permeability. Exp Lung Res 2016; 42: 380-395.

29 Midulla F, Nicolai A, Ferrara M, et al. Recurrent wheezing 36 months after bronchiolitis is associated with rhinovirus infections and blood eosinophilia. Acta Paediatr 2014; 103: 1094-1099.

30 Bergroth E, Aakula M, Korppi M, et al. Post-bronchiolitis use of asthma medication: a prospective 1-year follow-up study. Pediatr Infect Dis J 2016; 35: 363-368.

31 Cox DW, Bizzintino J, Ferrari G, et al. Human rhinovirus species C infection in young children with acute wheeze is associated with increased acute respiratory hospital admissions. Am J Respir Crit Care Med 2013; 188: $1358-1364$

32 Bizzintino J, Lee WM, Laing IA, et al. Association between human rhinovirus C and severity of acute asthma in children. Eur Respir J 2011; 37: 1037-1042.

33 Sly PD, Boner AL, Bjorksten B, et al. Early identification of atopy in the prediction of persistent asthma in children. Lancet 2008; 372: 1100-1106.

34 Lukkarinen M, Koistinen A, Turunen R, et al. Rhinovirus-induced first wheezing episode predicts atopic but not nonatopic asthma at school age. J Allergy Clin Immunol 2017; 140: 988-995.

35 Jartti T, Smits HH, Bonnelykke K, et al. Bronchiolitis needs a revisit: distinguishing between virus entities and their treatments. Allergy 2019; 74: 40-52.

36 Sigurs N, Bjarnason R, Sigurbergsson F, et al. Asthma and immunoglobulin E antibodies after respiratory syncytial virus bronchiolitis: a prospective cohort study with matched controls. Pediatrics 1995; 95: 500-505.

37 Stein RT, Sherrill D, Morgan WJ, et al. Respiratory syncytial virus in early life and risk of wheeze and allergy by age 13 years. Lancet 1999; 354: 541-545.

38 Goksor E, Amark M, Alm B, et al. High risk of adult asthma following severe wheezing in early life. Pediatr Pulmonol 2015; 50: 789-797. 